\title{
ACUTE CALCIFIC BURSITIS AFTER ULTRASOUND-GUIDED PERCUTANEOUS BARBOTAGE OF ROTATOR CUFF CALCIFIC TENDINOPATHY
}

BoSung Kang ${ }^{1,2}$, Sun Gun Chung

'Department of Rehabilitation Medicine, Seoul National University Hospital, Korea ${ }^{2}$ Department of Rehabilitation, Sheikh Khalifa Specialty Hospital, UAE

\section{Introduction}

Rotator cuff calcific tendinopathy is one of common causes of shoulder pain. Ultrasound (US)-guided barbotage is regarded as an excellent treatment option.

$>$ Although clinical improvement after US-guided barbotage was found in 71 to $91 \%$ of patients, postprocedural complications including late painful bursitis have been reported. In most of these cases, US reevaluation revealed no macroscopic deposits requiring additional treatment.

$>$ In contrast, here we report a patient with acute postprocedural subacromial-subdeltoid (SASD) bursitis due to newly developed, massive intra-bursal calcification.

\section{Case Presentation}

$>$ A 49-year-old woman without any trauma history gradually developed bilateral shoulder pain during a period of one year, which aggravated progressively with a waxing and waning course despite supportive physical therapy and oral medications.

$>$ Radiography and ultrasonography of her shoulders revealed large, bilateral, oval-shaped calcific deposits in her supraspinatus tendons(SST). Because her right shoulder pain was more severe than her left shoulder pain, US-guided barbotage was first performed on her right SST. When her right shoulder pain resolved after two weeks, she underwent the same procedure for her left SST.

$>$ Three weeks after the left shoulder procedure the patient returned with severe left shoulder pain contrary to right shoulder where pain resolved. Follow up radiography and ultrasonography showed little SST calcification; however, massive calcification in the left SASD bursa was discovered, indicating severe calcific bursitis.

$>$ Therefore, we performed US-guided barbotage targeted for calcific materials in left SASD bursa using the same protocol. After barbotage, a larger volume of normal saline $(12 \mathrm{ml})$ with triamcinolone $(40 \mathrm{mg})$ was injected into the SASD bursa this time. Three weeks later, she had no complaints about pain or limited range of shoulder motion. Follow up ultrasonography and radiography showed decreased SST and SASD bursa calcification.

\section{Conclusion}

We present an acute calcific SASD bursitis following barbotage. A high index of suspicion is required in patients with unresolved or aggravated pain after barbotage, which can be effectively treated by additional procedures.

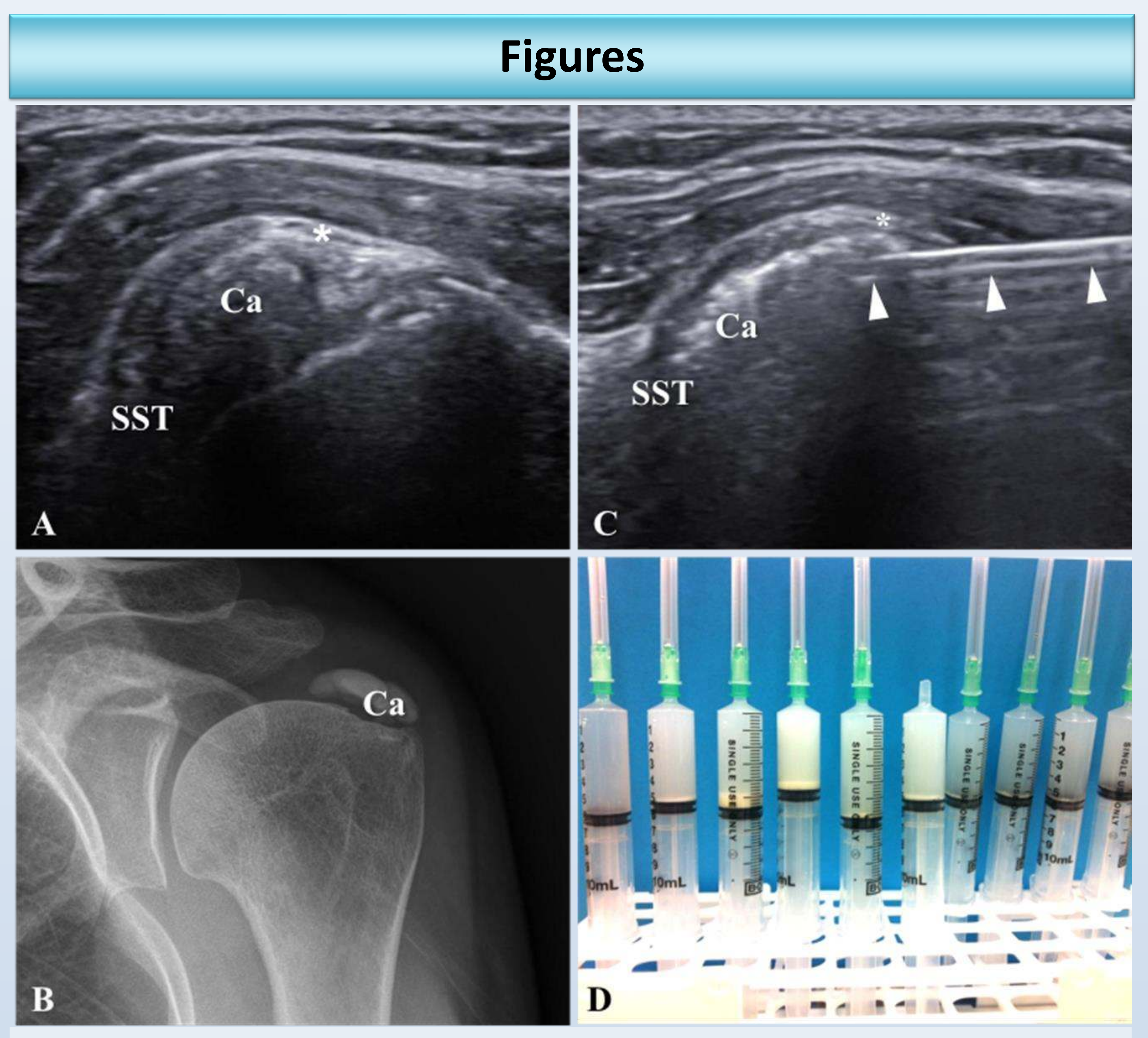

(A) Ultrasonography and (B) plain radiography show calcification (Ca) of left SST. The size of calcification is $22.3 \times 5.5 \mathrm{~mm}$. (C) The tip of an 18gauge spinal needle is thrust through the bursal surface of the tendon into the calcific deposit, under ultrasound-guidance. (D) Cloudy calcific material is removed from the SST by the syringe.
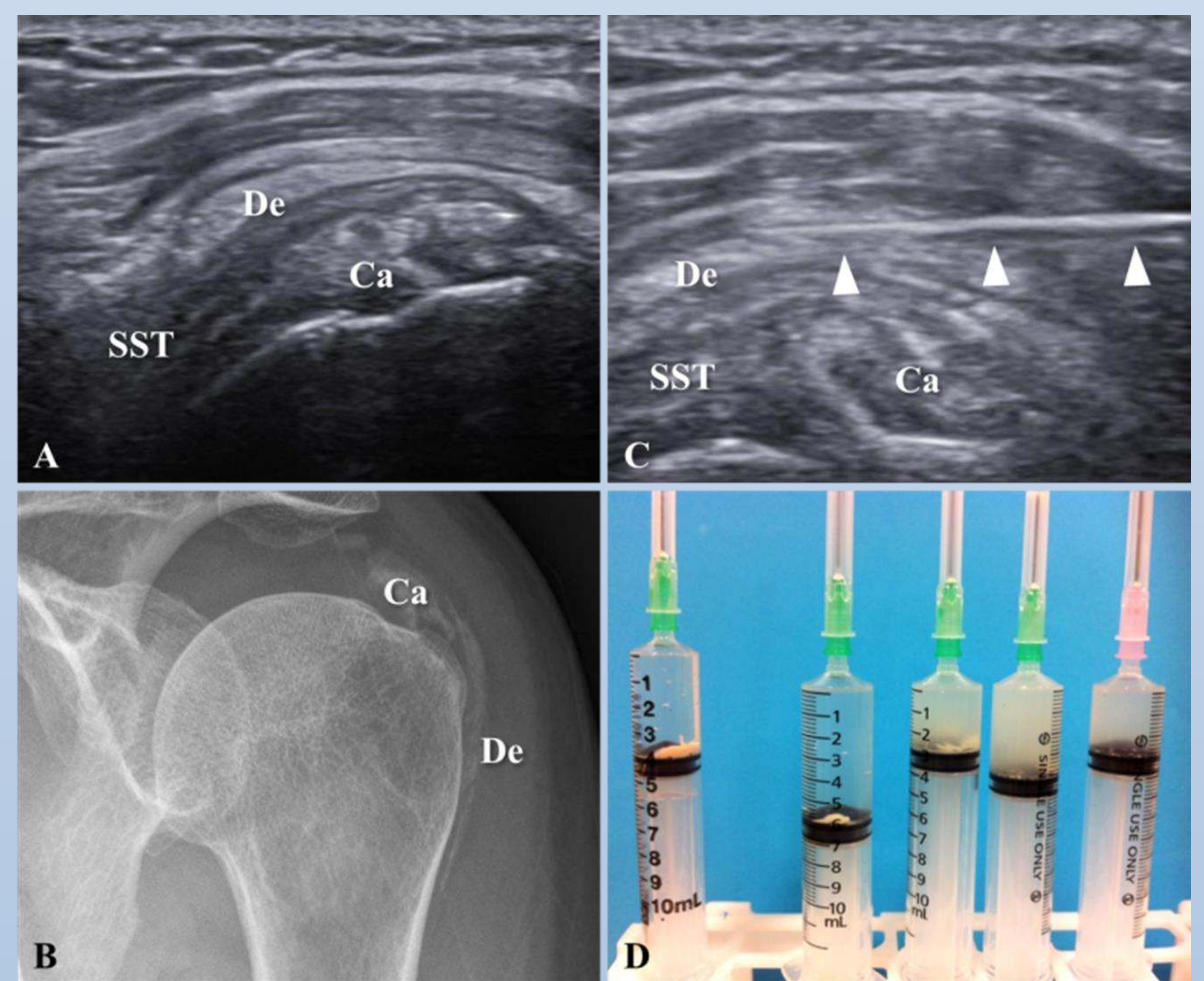

B

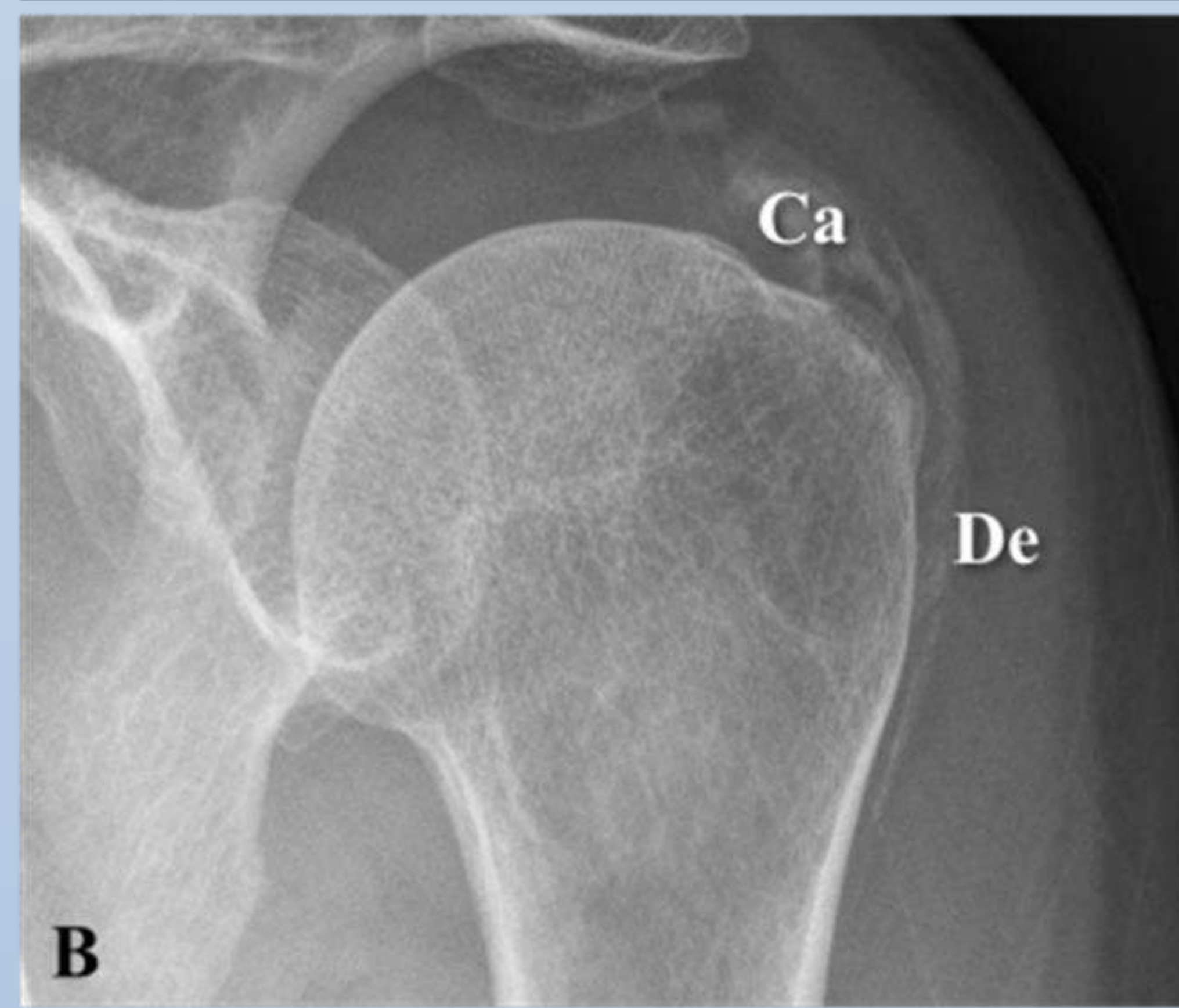

D

(A) Ultrasonography and (B) plain radiography show thick calcific deposits (De) spread along the SASD. Some calcification (Ca) remained in the SST. (C) The tip of an 18-gauge spinal needle is inserted into the SASD calcification under ultrasound-guidance. (D) The amount of calcific material drained from the SASD bursa is considerable, but less than that drained from the SST.

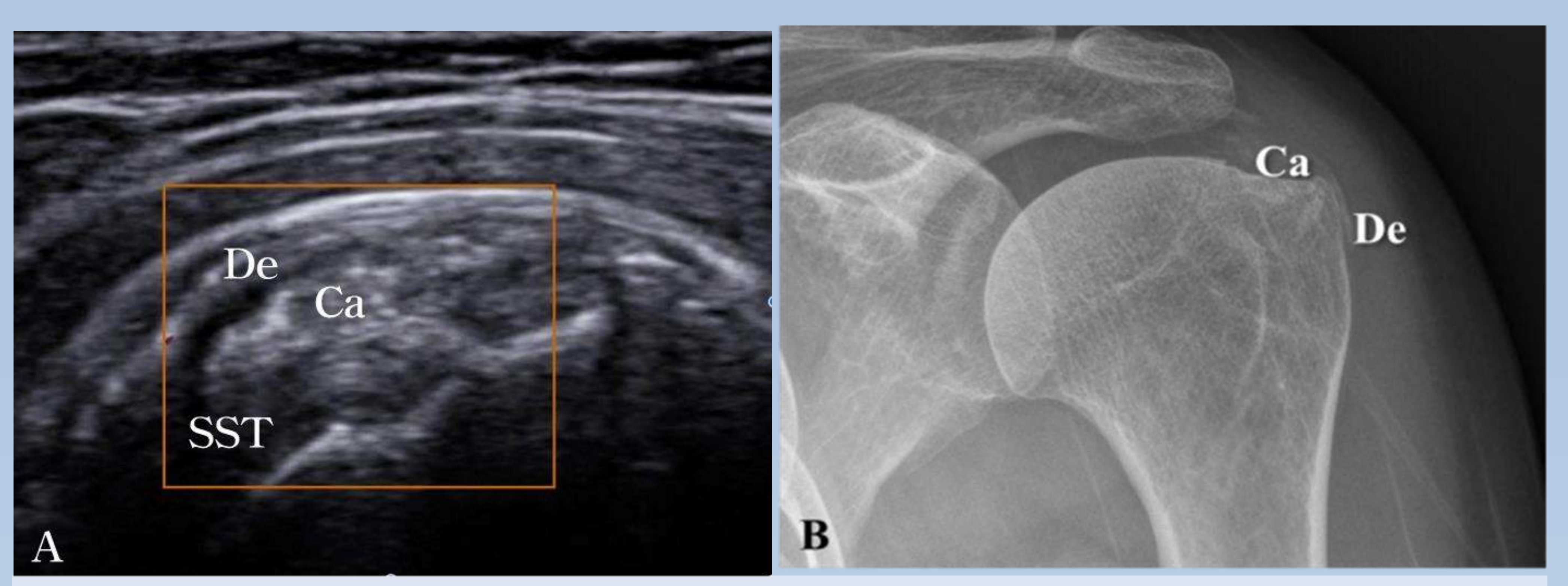

(A) Ultrasonography and (B) plain radiography show substantially decrease d calcific deposits ( $\mathrm{Ca}$ ) in SST and SASD bursa (De). 